\title{
Water Microsolvation Can Switch the Binding Mode of Ni(II) with Small Peptides
}

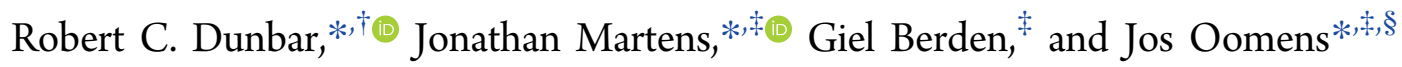 \\ ${ }^{\dagger}$ Chemistry Department, Case Western Reserve University, Cleveland, Ohio 44106, United States \\ ${ }^{\ddagger}$ Radboud University, Institute for Molecules and Materials, FELIX Laboratory, Toernooiveld 7c, 6525ED Nijmegen, The \\ Netherlands \\ ${ }^{\S}$ University of Amsterdam, Science Park 904, 1098XH Amsterdam, The Netherlands
}

\section{Supporting Information}

ABSTRACT: $\mathrm{Ni}(\mathrm{II})$ ions can be caged by surrounding peptide ligands in two basic binding patterns: the "iminol" (IM) binding pattern, where chelation occurs by deprotonated amide nitrogens, or the charge-solvated (CS) binding pattern, where chelation occurs by amide carbonyl oxygens. Gas-phase observation may clarify the factors affecting this choice in solution and in peptide and protein matrices. Infrared spectroscopic determination of gas-phase structures shows here how microsolvation by just one water molecule switches the balance of this choice from IM to CS for the $\mathrm{Ni}^{2+} \mathrm{Gly}_{3}$ complex, in contrast with the always-CS structure of the $\mathrm{Ni}^{2+} \mathrm{Gly}_{4}$ complex. Quantum-chemical calculations indicate that CS complexation is even more favored in the aqueous limit. Considering gas-phase conditions as comparable to low-pH solutions can reconcile this prediction with the common observation of IM-type binding in solutions at higher $\mathrm{pH}$. This is likely the first gas-phase observation of solvation-induced IM-to-CS transition in oligopeptide complexes with doubly charged transition-metal ions.

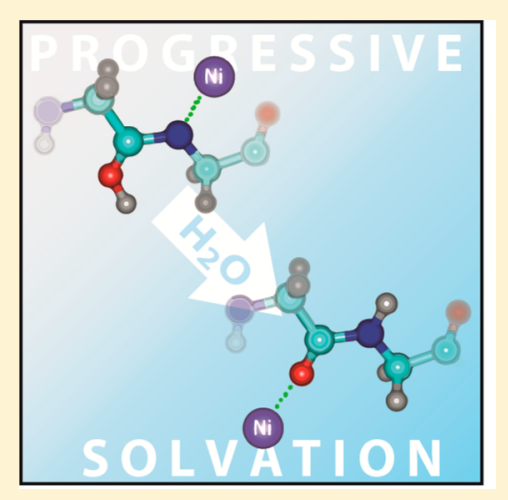

A $\mathrm{n}$ intriguing aspect of the chemistry of complexes of metal ions with amino acids and small peptides is the frequent change in conformation of the peptide ligand upon drying out the aqueous-phase complex and introducing it into the gas phase. It is interesting to consider the determinative role of the surroundings of the complex, whether it be gas-phase vacuum, aqueous solvation, or inclusion in the interior of a protein or enzyme. The emergence in the last decades of powerful tools for determination of the structures of gas-phase complexes has stimulated consideration of the gas-phase environment. One approach to such understanding addresses cases where the passage from the bare complex to the aqueous solvated complex through progressive attachment of water molecules can be pivotal in the choice of structure.

One common structure transition that has received much attention in recent gas-phase studies is charge-solvation (CS) binding (involving the canonical form of the ligand) versus zwitterionic structures (salt-bridge-stabilized (SB) binding, leading to the ammonium zwitterion form of the ligand). ${ }^{1-3}$ Examined in the present study is another class of structural alternatives possibly transiting between solution and the gas phase: the choice of metal-ion chelation by a cage of Lewisbasic sites, predominantly amide backbone carbonyl oxygens (Chart 1 , structures 1 and 2), versus a square-planar cage involving metal-nitrogen bonds derived from deprotonated amide backbone $\mathrm{NH}$ groups (Chart 1, structure 3 ).

Although much studied in solution with transition-metal ions, ${ }^{4}$ the latter complexation pattern through deprotonation of amide nitrogens has not been so widely examined for gas-phase

complexes. For gas-phase complexes of the metal ion with the neutral peptide (that is, not explicitly deprotonated in their gasphase form), the structures chelating the metal ion by deprotonated amide nitrogens can be accessed through iminol tautomerization of the initially canonical amide backbone residues. ${ }^{5}$ We designate as "charge solvated" (CS) the form of the complex having the metal chelated by amide carbonyl oxygens and as "iminol" (IM) the form of the complex resulting from iminol rearrangements to chelate the metal ion with deprotonated amide nitrogens. In solution-phase peptide complexes of transition metals like $\mathrm{Ni}(\mathrm{II})$ and $\mathrm{Cu}(\mathrm{II})$, a transition from CS- to IM-type binding is observed with increasing $\mathrm{pH}$. For polyglycine complexes of $\mathrm{Ni}(\mathrm{II})$, in particular, octahedral high-spin CS-type coordination converts to square-planar IM-type complexation with explicit deprotonation of the ligand near $\mathrm{pH} 8$ or $9 .^{4,6}$ In the gas phase, IM complexation has also frequently been reported for these metals, ${ }^{3,7,8}$ but CS complexation is favored in some cases. ${ }^{9}$ The choice between IM and CS complexation patterns has been of recent interest to our research group, ${ }^{7-10}$ and the influence of the first solvating water molecule on this choice is the subject of the present report.

As background for the present study, numerous computational and experimental studies have reported the effect of water on the CS-to-SB switch through water microsolvation,

Received: April 20, 2017

Accepted: May 24, 2017

Published: May 24, 2017 


\section{Chart 1. Illustrative Structures of Complexes}

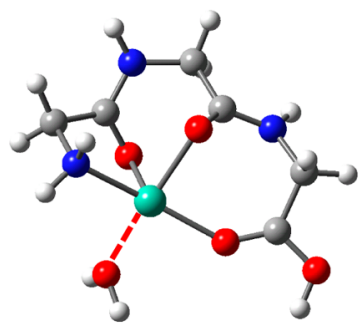

1

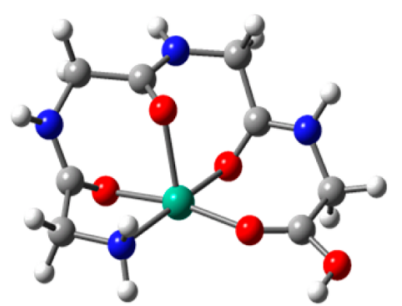

2

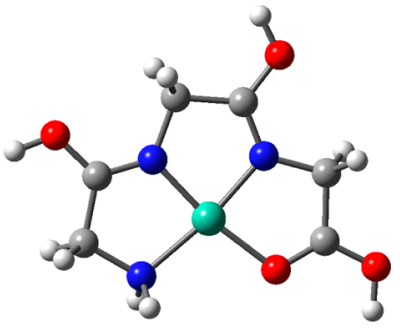

3 particularly for amino acids, ${ }^{1,1-15}$ including a few involving metal-ion complexes. ${ }^{16-20}$ The present report may be the first to characterize the CS-to-IM switch in small gas-phase metalion-peptide complexes.

Figure 1 displays the infrared multiple-photon dissociation (IRMPD) spectra of the $\mathrm{Gly}_{3}$ and $\mathrm{Gly}_{4}$ complexes with $\mathrm{Ni}^{2+}$,

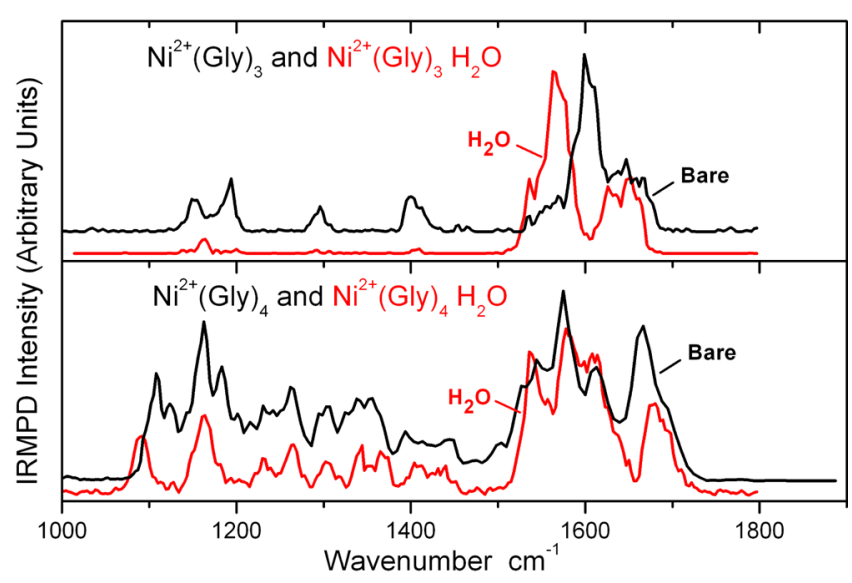

Figure 1. IRMPD spectra of the bare (black) and singly hydrated (red) complexes of $\mathrm{Ni}^{2+} \mathrm{Gly}_{3}$ and $\mathrm{Ni}^{2+} \mathrm{Gly}_{4}$.

with and without a single water molecule attached. For the $\mathrm{Gly}_{3}$ case, it is clear that water attachment induces a substantial structure change, looking, in particular, at the diagnostic region of the Amide I and Amide II modes between 1500 and 1650 $\mathrm{cm}^{-1}$. Similar results were found for the corresponding $\mathrm{Ala}_{3}$ system (Supporting Information, Figures S1 and S2). In the following, the spectra will be analyzed in detail, which will show that the attachment of the water molecule induces IM to CS rearrangement of the peptide. In contrast, the spectra of the $\mathrm{Gly}_{4}$ complex with and without $\mathrm{H}_{2} \mathrm{O}$, shown in the lower panel of Figure 1, are virtually identical, indicating that the water attachment does not induce a substantial change in the structure of the complex.

It remains to confirm, via comparison of the observed spectra with simulated spectra and strengthened by computed relative energies, that the structures of the $\mathrm{Gly}_{4}$ complexes are both CS, while the spectral shifts shown by the $\mathrm{Gly}_{3}$ complex are indeed due to a transition from IM to CS complexation.

Figure 2 shows the calculated structures and spectra for the most likely $\mathrm{Gly}_{3}$ structures, both bare and hydrated. Figure 3 shows similar results for the $\mathrm{Gly}_{4}$ complex. See the Supporting Information, Figure S3, for an additional spectrum of $\mathrm{Ni}^{2+} \mathrm{Gly}_{4}$. $\mathrm{H}_{2} \mathrm{O}$ in the $3000-3800 \mathrm{~cm}^{-1}$ spectral range, further confirming the CS structure of this monohydrate.

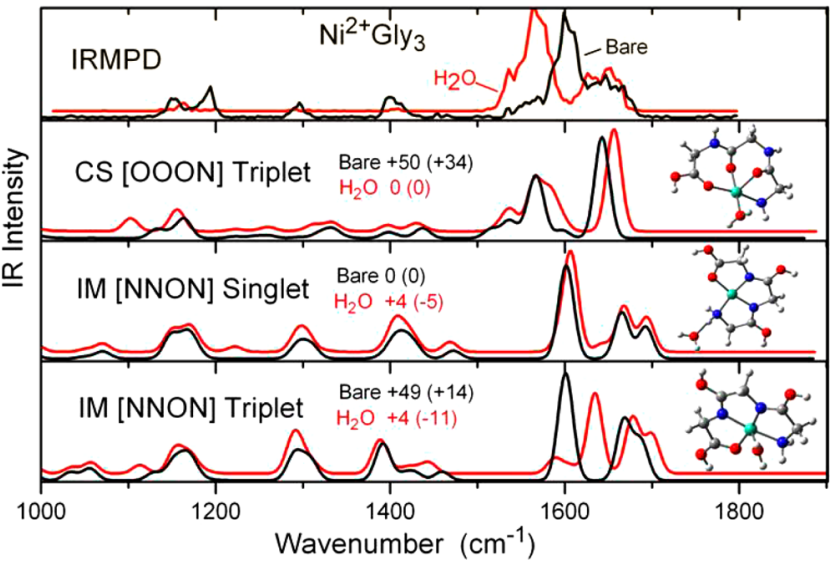

Figure 2. Observed (top panel) and computed (lower panels) spectra for the $\mathrm{Ni}^{2+} \mathrm{Gly}_{3}$ complex. Spectra for bare (black) and monohydrated (red) complexes are superimposed. Structures shown are for the monohydrates. The structure of the bare complex in all cases is close to the structure shown, minus the water molecule, but see the Supporting Information for larger pictures and coordinates of all six species. The notation [XYZ] indicates atoms bound to the metal; see ref 9 for notation details. Energies for bare and for monohydrated complexes relative to the most stable isomer are shown individually $(\mathrm{kJ}$ $\mathrm{mol}^{-1}$ ), predicting the switch from IM singlet to CS triplet ground state upon the addition of one $\mathrm{H}_{2} \mathrm{O}$. Values in parentheses are freeenergy-corrected (rigid-rotor harmonic-oscillator approximation at 298 $\mathrm{K})$. Note that the two IM structures displayed are essentially the same except for the position of the water molecule and the nickel spin. The energies reported for the corresponding "bare" complexes differ because of the promotion energy from singlet to triplet nickel.

In Figures 2 and 3, structures are drawn for the monohydrates. Note that the four-coordinate, singlet IM complexes hydrate on the exterior, while the triplet CS conformations hydrate directly on the nickel ion. Breaking up the favorable low-spin square planar cage of the IM nickel ion upon attachment of a water molecule always incurs a large energy cost, but this cost can be compensated for by the increased number of metal-ligand bonds possible for the CS binding mode accompanied by a switch from singlet to triplet nickel. Because a limit of six coordination points is not exceeded for any of the triplet CS conformations considered here, there is an energetic advantage to adding a water molecule onto the metal center to approach octahedral CS coordination.

All of the observed and calculated CS complexes have in common the predicted strong vibrational bands due to an antisymmetric COO stretch of the C-terminus at 1650-1700 $\mathrm{cm}^{-1}$, a group of mostly Amide I type vibrations around 1600 $\mathrm{cm}^{-1}$, and strong Amide II vibrations near $1550 \mathrm{~cm}^{-1}$. 


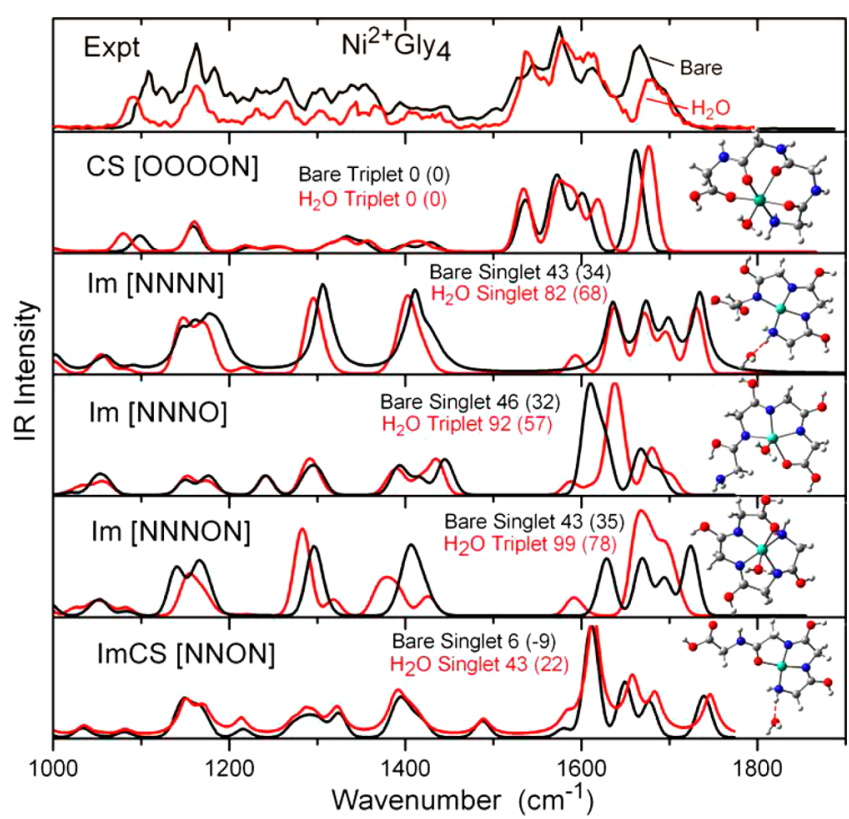

Figure 3. Observed and calculated spectra for the $\mathrm{Ni}^{2+} \mathrm{Gly}_{4}$ complex. Bare (black) and monohydrated (red) are superimposed. Structures shown are for the monohydrates; the structure of the bare complex is close to the structure shown, minus the water molecule, except IM [NNNON]. See the Supporting Information for larger pictures and coordinates of all ten species. The bare complexes of IM [NNNN] and IM [NNNON] are actually the same structure because they both dehydrate to give the same species. Relative calculated energies in $\mathrm{kJ}$ $\mathrm{mol}^{-1}$ with free energies at $298 \mathrm{~K}$ are given in parentheses.

The IM structures ([NNON] for Gly3; [NNNO], [NNNON], [NNNN], and IMCS for $\mathrm{Gly}_{4}$ ) are all built upon the square-planar coordination pattern. The low-spin (singlet) state is normally favored over the triplet for a given IM conformation, although in some cases the square-pyramidal triplet is accessible at a reasonable energy cost. In the 1600$1750 \mathrm{~cm}^{-1}$ range the IM-type structures exhibit a strong carboxyl mode and two or three $\mathrm{C}-\mathrm{N}$ stretching-dominated modes derived from the amide linkages as well as clusters of $\mathrm{CH}$ bending modes at $1400-1450$ and $1300-1350 \mathrm{~cm}^{-1}$. The most useful for structure characterization is the presence (CS) or absence (IM) of Amide II intensity near $1550 \mathrm{~cm}^{-1}$. The calculated spectra give very acceptable agreement with the assigned structures for both the bare and the hydrated complexes.

It is interesting to consider the trend of energies as hydration is increased beyond monohydration and especially in the limit of fully hydrated (that is, aqueous) complexes. Experimental examination of these complexes in the solution phase is not foreseen, but theoretical computation for full water solvation may be approximated using the SCRF (self-consistent reaction field) approach. Table 1 summarizes the energy trends from the bare complex to the monohydrate to full solvation. Two IM reference calculations are presented for the Gly 4 case: The $\mathrm{Ni}^{2+}\left(\mathrm{Gly}_{4}\right)$ IMCS result refers to the most stable IM-type conformation, which is the mixed IMCS [NNON] structure. However, using IMCS as a reference for comparison with solution phase may be misleading because the strong hydrogen bond remote from the metal site in the gas-phase IMCS complex (see Figure 3) probably does not provide similar stabilization in solution. Accordingly, the structure IM [NNNN] having three dehydrated amide nitrogens binding the metal is also shown as an example of a possibly more realistic reference for comparison with solution phase.

For $\mathrm{Gly}_{3}$, monohydration substantially increases the relative stability of CS, and infinite hydration (SCRF) even more so. This progressive increase in CS stability with hydration may come from relieving the coordinative unsaturation of the lessthan-octahedral metal ion in triplet CS versus the coordinative saturation in the square-planar singlet IM complex. Similarly, monohydration of $\mathrm{Gly}_{4}$ gives an increase in relative CS stability, and infinite hydration appears to give at least some CS stabilization. Thus the CS conformations are predicted to be favored in solution as well as in gas phase, but these would probably only be observed at low $\mathrm{pH}$, where in practice other solution chemistry such as dimerization is found to intervene. The monomeric IM-type complexes are well known in solution but only at high $\mathrm{pH}^{4,6}$ We can take the point of view that the gas-phase conditions correspond to low- $\mathrm{pH}$ condensed-phase environments, where outright deprotonation of the system is not favored but rather displacement of the amide proton could occur in the iminol rearrangement comparable to the gas-phase situation.

In conclusion, the switch of conformation from IM (squareplanar singlet) to CS (pyramidal triplet) upon monohydration of the $\mathrm{Gly}_{3}$ complex is strongly confirmed by IRMPD spectroscopy, and the reversal in stability of the two structures upon monohydration is further affirmed by the B3LYP thermochemical calculations. The $\mathrm{Gly}_{4}$ complex offers a direct contrast in that the structure of the $\mathrm{Gly}_{4}$ complex is confirmed by the spectroscopy to be CS for both the bare complex (pyramidal) and the monohydrate (octahedral).

A simple qualitative argument can account for these results, similar to the ligand-counting argument in ref 9. It can be postulated that low-spin, square-planar four-coordination with IM metal-nitrogen bonds is better than high-spin fourcoordination with CS metal-oxygen bonds but worse than five-fold or higher CS coordination. Then, $\mathrm{Ni}^{2+} \mathrm{Gly}_{3}$, having at most four-coordination, prefers $\mathrm{IM}$, but $\mathrm{Ni}^{2+} \mathrm{Gly}_{3} \cdot \mathrm{H}_{2} \mathrm{O}$ with five-coordination prefers CS. Similarly, $\mathrm{Ni}^{2+} \mathrm{Gly}_{4}$ and $\mathrm{Ni}^{2+} \mathrm{Gly}_{4}$. $\mathrm{H}_{2} \mathrm{O}$, with five- and six-coordination, respectively, both prefer the CS pattern.

Table 1. Energy Advantage of CS Complexation (Best CS Structure) Relative to the IM Structure Specified in the Left-Hand Column in $\mathrm{kJ} \mathrm{mol}^{-1 a}$

$\begin{array}{lcccc} & \text { bare } & \text { monohydrate } & \text { SCRF }\left(\text { no H}_{2} \mathrm{O}\right) & \text { SCRF }\left(\text { one } \mathrm{H}_{2} \mathrm{O}\right) \\ \mathrm{Ni}(\mathrm{II})\left(\mathrm{Gly}_{3}\right) \text { IM }[\mathrm{NNON}] & -50(-34) & +4(-5) & +31(+12) & +40(+24) \\ \mathrm{Ni}(\mathrm{II})\left(\mathrm{Gly}_{4}\right) \text { IMCS }[\mathrm{NNON}] & +6(-9) & +43(+22) & +13(-3) & +23(-7) \\ \mathrm{Ni}(\mathrm{II})\left(\mathrm{Gly}_{4}\right) \text { IM }[\mathrm{NNNN}] & +43(+35) & +82(+69) & +54(+44) & +76(+49)\end{array}$

${ }^{a}$ Values in parentheses are free-energy-corrected in the rigid-rotor harmonic-oscillator approximation at $298 \mathrm{~K}$. Positive values indicate that CS is more stable than IM. 


\section{METHODS}

Computational. All calculations were carried out using the Gaussian09 quantum chemical package. ${ }^{21}$ Density functional theory (DFT) calculations at the B3LYP/6-31+G(d,p) level served as the default computational methodology. Energies reported are corrected for vibrational zero-point energy, and values corrected to free energies at $298 \mathrm{~K}$ are also reported. Important energy comparisons were checked using the larger 6$311++G(d, p)$ basis set, which was also used for all values in Table 1. Calculated IR spectra were found to not depend strongly on basis set size; therefore, we have primarily used the smaller basis set for the spectra.

For comparison with IRMPD spectra, the computed harmonic frequencies were scaled by a factor of $0.965(0.975$ for the larger basis set, 0.955 in the $\mathrm{H}$-stretching region), consistent with recent work on small peptide systems at these levels of theory. ${ }^{9,22}$ Computed spectra were convoluted with a $20 \mathrm{~cm}^{-1}$ fwhm Gaussian line shape function. Solvation calculations used the SCRF method and a dielectric constant of 78 .

Experimental IRMPD Spectroscopy. IR spectra of the gaseous metal ion-peptide complexes were recorded employing a modified quadrupole ion trap mass spectrometer (Bruker, Amazon Speed ETD) coupled to the Free Electron Laser for Infrared eXperiments (FELIX), as has been detailed elsewhere. $^{23,24}$ Metal-ion peptide complexes were generated by electrospray ionization from solutions containing the peptide and metal salt $(1 \mu \mathrm{M}$ of each $)$ in acetonitrile $/ \mathrm{H}_{2} \mathrm{O}(\sim 1: 1)$. Target ions were trapped, mass-selected, and irradiated with the wavelength-tunable infrared light from FELIX. DFT-computed linear absorption IR spectra of candidate ion structures were compared with the observed spectra for structure assignment, with additional guidance from the computed thermochemistry.

\section{ASSOCIATED CONTENT}

\section{S Supporting Information}

The Supporting Information is available free of charge on the ACS Publications website at DOI: 10.1021/acs.jpclett.7b00973. Spectra of corresponding $\mathrm{Ni}^{2+} \mathrm{Ala}_{n}$ complexes; OPO spectrum for $\mathrm{Ni}^{2+} \mathrm{Gly}_{4} \cdot \mathrm{H}_{2} \mathrm{O}$; full citation for Frisch et al., ref 21; and optimized coordinates and large pictures of all the species mentioned in the calculations. (PDF)

\section{AUTHOR INFORMATION}

\section{Corresponding Authors}

*R.C.D.: E-mail: rcd@po.cwru.edu.

*J.M.: E-mail: jonathan.martens@science.ru.nl.

*J.O.: E-mail: joso@science.ru.nl.

ORCID $\odot$

Robert C. Dunbar: 0000-0002-5459-5527

Jonathan Martens: 0000-0001-9537-4117

\section{Notes}

The authors declare no competing financial interest.

\section{ACKNOWLEDGMENTS}

This work was financially supported by the "Nederlandse Organisatie voor Wetenschappelijk Onderzoek" (NWO) under vici-grant no. 724.011.002. R.C.D. acknowledges support from the National Science Foundation, Grant PIRE-0730072, and expresses gratitude to the FELIX facility for its continuing welcome. The FELIX staff and operators are gratefully acknowledged for their excellent assistance. We thank SURFsara Computing and Networking Services (www. surfsara.nl) for their support in using the Lisa Compute Cluster and NWO Physical Sciences (EW) for providing access under grant no. 15408. We gratefully acknowledge Stichting voor Fundamenteel Onderzoek der Materie (FOM) for the support of the FELIX Laboratory.

\section{REFERENCES}

(1) Wyttenbach, T.; Bowers, M. T. Hydration of biomolecules. Chem. Phys. Lett. 2009, 480, 1-16.

(2) Burt, M. B.; Fridgen, T. D. Structures and physical properties of gaseous metal cationized biological ions. Eur. Mass Spectrom. 2012, 18, 235-250.

(3) Dunbar, R. C. Spectroscopy of Metal-Ion Complexes with Peptide-Related Ligands. Top. Curr. Chem. 2014, 364, 183-223.

(4) Sovago, I.; Osz, K. Metal Ion Selectivity of Oligopeptides. Dalton Trans. 2006, 3841-3854.

(5) Dunbar, R. C.; Steill, J. D.; Polfer, N. C.; Berden, G.; Oomens, J. Peptide Bond Tautomerization Induced by Divalent Metal Ions: Characterization of the Iminol Configuration. Angew. Chem., Int. Ed. 2012, 51, 4591-4593.

(6) Martin, R. B. Nickel Ion Binding to Amino Acids and Peptides. In Metal Ions in Biological Systems: Nickel and Its Role in Biology; Sigel, A., Sigel, H., Eds.; Marcel Dekker: New York, 1988; Vol. 23, pp 123-164.

(7) Dunbar, R. C.; Berden, G.; Oomens, J. How Does a Small Peptide Choose How to Bind a Metal Ion? IRMPD and Computational Survey of CS Versus Iminol Binding Preferences. Int. J. Mass Spectrom. 2013, 354-355, 356-364.

(8) Dunbar, R. C.; Polfer, N. C.; Berden, G.; Oomens, J. Metal Ion Binding to Peptides: Oxygen or Nitrogen Sites? Int. J. Mass Spectrom. 2012, 330-332, 71-77.

(9) Dunbar, R. C.; Martens, J.; Berden, G.; Oomens, J. Complexes of $\mathrm{Ni}(\mathrm{II})$ and $\mathrm{Cu}(\mathrm{II})$ with small peptides: deciding whether to deprotonate. Phys. Chem. Chem. Phys. 2016, 18, 26923-26932.

(10) Peckelsen, K.; Martens, J.; Berden, G.; Oomens, J.; Dunbar, R. C.; Meijer, A.; Schäfer, M. Gas-phase complexes of $\mathrm{Ni2}+$ and $\mathrm{Ca} 2+$ with deprotonated histidylhistidine (HisHis): A model case for polyhistidyl-metal binding motifs. J. Mol. Spectrosc. 2017, 332, 38-44.

(11) Blom, M. N.; Compagnon, I.; Polfer, N. C.; von Helden, G.; Meijer, G.; Suhai, S.; Paizs, B.; Oomens, J. Stepwise solvation of an amino acid: The appearance of zwitterionic structures. J. Phys. Chem. A 2007, 111, 7309-7316.

(12) Kim, J. Y.; Lee, Y.; Lee, S. Effects of microsolvation on the relative stability of zwitterionic vs. canonical proline. Chem. Phys. Lett. 2014, 608, 177-185.

(13) Lee, S. S.; Kim, J. Y.; Han, Y.; Shim, H. J.; Lee, S. Thermodynamic and kinetic stability of zwitterionic histidine: Effects of gas phase hydration. Chem. Phys. Lett. 2015, 637, 42-50.

(14) Pathak, A. K. Stabilizing the zwitter-ionic form of amino acids in the gas phase: An ab initio study on the minimum number of solvents and ions. Chem. Phys. Lett. 2014, 610, 345-350.

(15) Prell, J. S.; Chang, T. M.; O’Brien, J. T.; Williams, E. R. Hydration Isomers of Protonated Phenylalanine and Derivatives: Relative Stabilities from Infrared Photodissociation. J. Am. Chem. Soc. 2010, 132, 7811-7819.

(16) Moghaddam, M. B.; Fridgen, T. D. IRMPD Spectroscopic Study of Microsolvated $[\mathrm{Na}($ GlyAla $)]+$ and $[\mathrm{Ca}($ GlyAla-H $)]+$ and the Blue Shifting of the Hydrogen-Bonded Amide Stretch with Each Water Addition. J. Phys. Chem. B 2013, 117, 6157-6164.

(17) Gholami, A.; Fridgen, T. D. Structures and Unimolecular Reactivity of Gas-Phase $[\mathrm{Zn}($ Proline- $\mathrm{H})]+$ and $[\mathrm{Zn}($ Proline- $\mathrm{H})-$ (H2O)]+. J. Phys. Chem. B 2013, 117, 8447-8456.

(18) Remko, M.; Fitz, D.; Rode, B. M. Effect of metal ions ( $\mathrm{Li}+, \mathrm{Na}+$, $\mathrm{K}+, \mathrm{Mg} 2+, \mathrm{Ca} 2+, \mathrm{Ni} 2+, \mathrm{Cu} 2+$, and $\mathrm{Zn} 2+)$ and water coordination on the structure and properties of L-arginine and zwitterionic L-arginine. J. Phys. Chem. A 2008, 112, 7652-7661. 
(19) Bush, M. F.; Prell, J. S.; Saykally, R. J.; Williams, E. R. One water molecule stabilizes the cationized arginine zwitterion. J. Am. Chem. Soc. 2007, 129, 13544-13553.

(20) Lemoff, A. S.; Bush, M. F.; Williams, E. R. Structures of cationized proline analogues: evidence for the zwitterionic form. J. Phys. Chem. A 2005, 109, 1903-1910.

(21) Frisch, M. J.; et al. Gaussian 09, revision A.02; Gaussian, Inc.: Pittsburgh, PA, 2009. See the Supporting Information for full reference.

(22) Wu, R. R.; Yang, B.; Berden, G.; Oomens, J.; Rodgers, M. T. Gas-Phase Conformations and Energetics of Protonated 2'-Deoxyguanosine and Guanosine: IRMPD Action Spectroscopy and Theoretical Studies. J. Phys. Chem. B 2014, 118, 14774-14784.

(23) Martens, J.; Grzetic, J.; Berden, G.; Oomens, J. Structural identification of electron transfer dissociation products in mass spectrometry using infrared ion spectroscopy. Nat. Commun. 2016, $7,11754$.

(24) Martens, J.; Berden, G.; Gebhardt, C. R.; Oomens, J. Infrared ion spectroscopy in a modified quadrupole ion trap mass spectrometer at the FELIX free electron laser laboratory. Rev. Sci. Instrum. 2016, 87, 103108. 